\title{
Thomas Mann: Der Zauberberg
}

Ida Boy-Ed

Follow this and additional works at: https://scholarsarchive.byu.edu/sophnf_essay

Part of the German Literature Commons

\section{BYU ScholarsArchive Citation}

Boy-Ed, Ida, "Thomas Mann: Der Zauberberg" (1975). Essays. 763.

https://scholarsarchive.byu.edu/sophnf_essay/763

This Article is brought to you for free and open access by the Nonfiction at BYU ScholarsArchive. It has been accepted for inclusion in Essays by an authorized administrator of BYU ScholarsArchive. For more information, please contact scholarsarchive@byu.edu, ellen_amatangelo@byu.edu. 


\section{Thomas Mann: Der Zauberberg}

Die politische Stellung eines Schaffenden soll bei der Betrachtung seines Werkes sich nicht in das Bewußtsein drängen. In Zeiten, die so von Unruhe durchströmt sind wie die Gegenwart, wird es sich nie ganz vermeiden lassen, daß die Farbe der Gesinnung des Autors in seinem Werk doch, wenn auch nur sehr übertragen, erkennbar wird. Unter besonderen Fügungen aber, die nur vom Künstler selbst herbeigeführt oder gar provoziert werden können, ist es nicht möglich zu übersehen, wo er in der Politik steht. Solcher Fall tritt ein, wenn das Werk einem Parteizweck propagandorisch dienen soll, oder wenn der Künstler sich außerhalb seines Werks in der Politik betätigt unter jäher und auffallender Änderung seines vorherigen Standpunktes. Solche Änderung ist Thomas Manns Geste gewesen. Er hat die Peinlichkeit auf sich genommen, sich selbst zu widersprechen. Er, der in seinem großen Werk "Betrachtungen eines Unpolitischen « Wagners Satz: »Die Demokratie ist in Deutschland ein durchaus übersetztes Wesen. Sie existiert nur in der Presse«, einen unsterblichen und erlösenden Satz nannte; er, der im gleichen Buch feststellte, daß in Deutschland die Bejahung des Nationalen die Verneinung der Politik und der Demokratie in sich schließe, der sich mit Stolz als »Bürger « bezeichnete, ist Demokrat geworden; er, der den Begriff des Zivilisationsliteraten überhaupt erst schuf, hat sich neben ihn gestellt. So kam er nun in eine ähnliche Lage, wie etwa die Generale v. Deimling und v. Schönaich, die noch Titel und Ehren weiterführen, die ihnen unter anderen politischen und ethischen Bedingungen verliehen wurden. Nun, das ist seine Sache (soweit jemand, der der Öffentlichkeit durch sein Wirken angehört, ganz unbedingt von "seiner « Sache sprechen darf - was vielleicht zu verneinen, aber einer $\mathrm{zu}$ weitläufigen Begründung bedürfte, um hier untersucht werden zu können).

Meine Sache aber war es mit der vollen Aufrichtigkeit, die unter Charakteren, die sich seit Jahren in Hochachtung und Sympathie begegneten, selbstverständlich ist, an Thomas Mann redlichen Herzens zu schreiben, daß seine politische Wandlung mich schmerzt. Er hat diese Mitteilung mit der Würde des bedeutenden Mannes aufgenommen, der Offenheit schätzt (nur kleine Geister vertragen sie nicht!) und er hofft, daß die Zeit seine Stellung erklärlich machen werde und daß sein Werk »Der Zauberberg « dafür schon manche Vorarbeit leiste. (Ehe ich an die Betrachtung dieses Buches gehe, muß ich noch eine Paranthese mir gönnen: immer kristallener und imposanter erscheint Goethes, ein Menschenalter lang 
als kalt und unnational charakterisierte Haltung, fern und hoch über den Evolutionen seiner Gegenwart!)

Also nun »Der Zauberberg«. In Kürze kann kein Referent über diese Arbeit sprechen. Schriftleiter und Leser müssen mir Ausführlichkeit gestatten. Eine Äußerlichkeit sei vorweggenommen: die beiden starken Bände umfassen zusammen 1207 Seiten in leider sehr blasser, kleiner Schrift. Vielleicht hängt das mit der Einteilung der Gesamtausgabe zusammen. Oder der Verlag wollte vielleicht vermeiden, daß das Buch als ein vierbändiges Werk in unsere zerpeitschte Atmosphäre hinausträte. Warum nicht? Diese monumentale Arbeit richtet sich nur an höchst Kultivierte und sie würden das geistige Leben in und mit dem Werk leichter haben, wenn es zu ihnen in vier schlanken Bänden und ausgeprägtem Druck gekommen wäre.

Zwei Aussprüche kann ich über meinen Bericht setzen. Der eine stammt von Thomas Mann selbst (S. 59 I) »Zu bedeutender, das Maß des schlechthin Gebotenen überschreitender Leistung aufgelegt zu sein, ohne daß die Zeit auf die Frage >Wozu?` eine befriedigende Antwort wüßte, dazu gehört eine sittliche Einsamkeit und Unmittelbarkeit, die selten vorkommt und heroischer Natur ist. "Stärker, treffender und feierlicher kann die Arbeit des geistig Schaffenden nicht gewürdigt werden! Die ganze erschütternde Hingabe an das Werk ist damit ausgesprochen, welcher Hingabe gegenüber nur die vage Unsicherheit eines möglichen Verständnisses und Erfolges steht. Und wenn wir so den "Zauberberg«, im charakterisierten Sinn, als eine Arbeit heroischer Natur bewundern müssen, erwächst aus dieser - ich möchte sagen: demütigen Haltung zugleich die Pflicht, alle Einwände gegen dieses Werk deutlich zu begründen. Und diese Einwände können nicht anders als sehr ernst und vielleicht fundamental sein. - Die andere Äußerung ist von Schopenhauer: »Die Aufgabe des Romanschreibers ist nicht, große Vorfälle zu erzählen, sondern kleine interessant zu machen. "Von der Bestreitbarkeit dieses Ausspruches sehe ich hier ab, darf ihn aber auf die Kunst von Thomas Mann beziehen, das Detail so genau und so suggestiv vorzutragen, daß es zum Träger der Spannung wird. (Goethe forderte von jedem Buche Spannung: auch ein wissenschaftliches solle den Fachmann, an den es sich richtet, in Spannung versetzen. Bei uns ist die Bezeichnung "spannend « fast ein literarisches Schimpfwort geworden). Diese seine Kunst erreicht im »Zauberberg « einen Gipfel, der nicht mehr übersteigert werden darf ohne Gefahr. 
Die Inhaltsangabe des geräumigen Werkes ist sehr rasch gegeben: Ein junger Hanseat, Hans Castorp, ein Normalprodukt aus altangesehenem Hause, ohne rechte freudige Lebensenergie, soll nach bestandenem Ingenieurexamen drei Wochen ausspannen, und es liegt für ihn auf der Hand, diese Zeit zum Besuch bei seinem Stiefvetter Joachim zu benutzen, der seit drei Monaten in Davos sich im Sanatorium befindet. Dieser Stiefvetter will Leutnant werden und vor dem Eintritt in sein Regiment eine "feuchte Stelle« in seiner Lunge ausheilen. Von dem Augenblick an, wo Hans Castorp in die Umwelt des Sanatoriums eintritt, fühlen wir, daß sein Wesen sich in dieser Atmosphäre körperlicher Leiden, sittlicher Haltlosigkeit und übersteigerter Nervenreizbarkeiten auflösen und folgenschweren Umwandlungen unterliegen wird. Der Autor erweist sich als Experte in Tuberkulose. Wir erleben alle durch sie möglichen Todesformen: wir sehen neben den Türen der »Moribunden« (d.h. der dem Tode nicht mehr zu Entreißenden) die Flaschen mit Sauerstoff stehen; wir spüren in den Brusttaschen der Patienten das Aufnahmeglas für Sputum; wir werden mit den Patienten bekannt, die einen Pneumathorax haben und unter sich in grausigem Humor den Verein »Halbe Lunge« bilden. Der empfängliche Leser wird zuletzt von einer Art Unruhe erfaßt, die ihn zwingen möchte, die eigene Temperatur zu messen. Es vibriert zwischen den Patienten ein schwüles, unverhülltes Interesse an den sexuellen Beziehungen der Einen zu den Andern und bei einem Skandal wettert der Chefarzt, der Hofrat Behrens, daß er nichts dafür könne, daß die Phtise nun mal mit besonderer Konkupiscenz verbunden sei. Mit unerhörter Meisterlichkeit wird dargestellt, wie aus dem harmlosen Besucher der Anstalt allmählich deren Klient wird, in einer Art dämonischer Rattenfängerschaft, zur Autosuggestion gebracht, auch ihm könne eine Kur hier oben Heilung kleiner Schäden bringen, von deren Dasein er bisher keine Ahnung hatte. Und aus der "kleinen Kur« wird dann ein Hängenbleiben, für Jahre, wenn nicht gar für immer. Die Patienten, sie solche Opfer sind, verlieren die Kraft zur unbekümmerten Existenz ohne den ganzen Heilapparat, sie verlieren jede innere Verbindung mit den Pflichten des Daseins.

Wir befinden uns also zwischen lauter Outsidern der Welt, und es ist schwer $\mathrm{zu}$ verstehen, welchen Gewinn es gerade in unserer Gegenwart bringen soll, außerhalb ihrer zu leben. Gerade jetzt, wo sie von jedem Menschen alle Kraft erfordert, den Untergang (nicht nur unseres Volkes) aufzuhalten! Der Begriff "Zeit « löst sich auf bei den Leidenden, die von der Kostbarkeit dieses unseres wertvollsten Besitzes (denn in ihr hat die 
Arbeit ihre Auswirkungsfähigkeit!) gar keine Vorstellung mehr haben. 158 Die geistreichen, kühnen oft, und oft spitzfindigen Gedankenspielereien, um diesen zerflatterten Begriff "Zeit« herum, sind verführerisch und bedenklich zugleich.

Hans Castorp, die Person, an deren Tageslauf jahraus, jahrein all diese furchtbaren Details der Leiden angereiht werden, wird vom Autor immer wieder als eine »einfache Natur«, als schlichter Jüngling betont. Aber, so durchaus Mann sich auch bemüht, den Vortrag Castorpscher Reden und Gegenreden jeder schönrednerischen Gewandtheit zu entkleiden: er hat doch Mannschen Geist, und zahllose seiner Randglossen, Einwürfe und eigenen Gedanken zeugen für seinen eingeborenen Intellekt. Ein »einfacher « Geist kann nicht Betrachtungen über das Leben anstellen, wie Hans (S. 468 u.w. I), nicht über die Zeit, nicht ein Wachtraumerlebnis haben, wie das im Schnee, nicht so verständnisvoll anatomische Offenbarungen in sich aufnehmen, vor allem aber nicht solch Verständnis für Menschen von ausnehmendem Format haben wie er. Hans Castorp ist nicht gesteigert, sondern erwacht! - Und was erlebt denn dieser junge Mann! Lauter Details.

All diese furchtbaren Details der erschreckendsten Leiden! Und natürlich sucht Thomas Mann eine endlose Reihe grotesker Gestalten zu Trägern dieser Leiden hervor. Seine Neigung, das Pathologische und Groteske darzustellen, ist von Gegnern zuweilen als Mangel an Ethik gedeutet. Das ist eine falsche Auffassung. In der tiefsten Wurzel aller Begabungen ist auch schon der Zwang ihrer Art und wohin sie wachsen will einbeschlossen. Und sollte nicht vielleicht, vielleicht sage ich, ein frühes und ahnungsschweres Empfinden für das Corruptible ihm eingeboren gewesen sein, 159 das in Teilen deutschen Bürgertums sich schleichend auszubreiten begann?

Nicht geleugnet werden soll, daß das Groteske leichter darzustellen ist, als das Schöne und es mag wohl sein, daß Thomas Mann die nützlichen Lehren, die uns Maupassant in seiner Vorrede $\mathrm{zu}$ »Pierre et Jean« gab, oft allzu sorgsam befolgt.

Mann hatte dies Werk schon vor dem Krieg begonnen: in seinen $\gg B e-$ trachtungen «, gelegentlich der Würdigung von Pfitzners Palestrina, spricht er davon und daß die Sympathie mit dem Tode ihn dabei bewege. Ob vor dem Kriege andere Bedingungen dies Werk empfangen hätten? Sozialhygienische Zwecke verfolgt es nicht. Ob ein dichterisches Werk aus ästhetischen Gründen alle diese Schilderungen vermitteln durfte, sei dahingestellt. Es spielt mit dem Grauen, es fantasiert groß, zynisch, poetisch - 
je nach dem Fall - über den Tod. Jetzt aber, nachdem der Krieg uns in eine unermeßliche Fülle der Leiden und des Todes riß, davon Millionen Herzen noch bluten, jetzt hätte er vielleicht besser das Werk noch unvollendet gelassen. Wie sich denn auch, in Folge der sich über Jahre hindehnenden Vorstellungskonzentrationen der Stoff an Details so gehäuft hat, daß der Verfasser nicht die letzte Selbstkritik der Beschränkung mehr besaß.

Wenn der erste Band der der Details genannt werden kann, dürfen wir den zweiten den der Gespräche nennen. Ohne daß es dem ersten Band an höchst bedeutenden Gesprächen und dem zweiten an erschrecklichen Details fehlt. Hans Castorp lernt in dem Italiener Settembrini eine Persönlichkeit von Temperament und hoher Intelligenz kennen. Dieser Mann ist »demokratischer Patriot«. »Er hat «, sagt Hans Castorp von ihm, »seine Bürgerpike am Altar der Menschheit geweiht, damit die Salami in Zukunft an der Brennergrenze verzollt werde. « Dieser Settembrini, der als Republikaner und Demokrat den Krieg verdammt, ist aber doch durchaus und jederzeit geneigt, für seines Vaterlands Vergrößerung gegen Wien zu marschieren. Neben diesem Republikaner, Freimaurer und schönrednerischem Pathetiker von würdigstem, liebevollstem Charakter, geht noch ein Jesuit jüdischer Herkunft von unerhörtem Wissen und scharfer Dialektik einher. Was diese beiden Männer vor und für Hans Castorp debattieren, um seine jugendliche Gefolgschaft gewissermaßen ringend, ist von wahrhaft blendendem Reichtum an Geist und Wissen. Ich glaube nicht, daß irgend ein anderer Mann in Deutschland, wissenschaftlicher oder schöngeistiger Provenienz, imstande wäre, sich in so funkelnder Sprache und mit so tiefgründiger Kenntnis über die vielfältigsten Kulturerscheinungen und Epochen zu ergehen, wie Mann es hier durch den Mund Settembrinis (wohl seines eigentlichen Überzeugungsträgers) und des Jesuiten Naphta tut: über Gothik, Musik, Republikanismus, Logenwesen, Zeitsinn, von der Mission des Proletariats, von Hygiene, Erziehung, Humanismus, Geschichte, Anatomie, Philosophie ... bald vom Standpunkt des Republikaners, bald von dem des Jesuiten aus. Und wenn die Gedankenfülle einen umkreist, wird man schließlich versucht, sich auf eine einfache Erkenntnis zurückzuziehen und sich darin $\mathrm{zu}$ resignieren, daß es keine ewigen Wahrheiten in all diesen Dingen gibt. Und ein gewisser Niederschlag der Betrachtungen will dann allerdings auch Thomas Manns politische Wandlung als vorübergehende - Unwichtigkeit festlegen! Einen sehr bemerkenswerten und sehr bestreitbaren Satz Settembrinis wollen wir hier 
doch abschreiben: "Die Literatur sei nichts anderes als eben dies: Die Vereinigung von Humanismus und Politik, welche sich um so zwangloser vollziehe, als ja Humanismus selber schon Politik und Politik Humanismus sei.« - Haben wir hier den »umgewandelten« Thomas Mann? - Hat er vergessen, daß die Geschichte uns auf zahllosen ihrer Blätter lehrt, daß Politik die Feindin des Humanismus war? - Und auch eine Äußerung Naphtas sei notiert: »Das Instinktive ist durchaus auf seiten des Nationalen und Gott selbst hat den Menschen den natürlichen Instinkt eingepflanzt, der die Völker veranlaßt hat, sich in verschiedenen Staaten voneinander zu sondern." -

Hans Castorp kann nicht ganz unberührt bleiben von der Atmosphäre der Sexualität, die durch das Sanatorium schwelt. Eine lang sich hinziehende Annäherung an eine fremdartige Frau, deren Freibrief die Krankheit ist, deren Herkunft aus jenseitigen Kaukasusländern ihr alle Ungebundenheit gibt, gipfelt endlich in einer knappen Stunde erfüllter Wünsche, aus der er - das Röntgenbild der Begehrten als Andenken hinwegnimmt, das Röntgenbild, das ihr feines Knochengerüst und ihr brüchiges Innere zeigt und das seine Phantasie mit dem zarten weißen Fleisch ihres Körpers umkleiden kann. Worauf er Jahr und Tag ihrer Wiederkehr wartet, um sie als Reisebegleiterin eines Mynheer Peeperkorn zurückkehren zu sehen. Die monumentale Außerordentlichkeit dieser zugleich grotesken und 162 dennoch Ehrfurcht einflößenden Gestalt lebensvoll hinzustellen, hat allerhöchste Kunst gefordert. Dieser Peeperkorn tötet sich, weil er sich als »Gottes Hochzeitsorgan empfand und das Versagen des Gefühls vor dem Leben als kosmische Katastrophe empfand « - so drückt Hans Castorp das aus, derselbe, den sein Autor einen »einfachen jungen Menschen« nennt! Dieser Tod zwingt Hans Castorp zu einem keuschen und zarten Abschied von der so lange Begehrten.

Und von da versinkt sein Leben in dem Stumpfsinn des Sanatoriums, den der Verfasser mit Recht »dämonisch« nennt, denn sein geheimes Wesen ist mörderisch. Alle Stadien leerer Scheinunterhaltungen, die die Geselligkeit kennt, werden durchlaufen und zuletzt muß die Beschäftigung mit dem Okkultismus die Patienten aufpeitschen; hier treffen wir auf einige Reminiszenzen aus dem uns bekannten Vortrag Manns über Okkultismus. Offen will ich sagen, daß man diese letzten Kapitel knapper gefaßt wünscht. Noch Bände lang könnte die Erzählung fortgesetzt werden, Detail an Detail reihend, wenn nicht jäh der Donnerschlag die Welt erschütterte der eine, der noch Jahrhunderte lang nachhallen wird ... 
Von einer Gestalt habe ich noch nichts gesagt, sie mit Vorsatz allein behandeln wollen. Das ist der junge Joachim, den zu besuchen Hans nach Davos reist, um dann seinerseits dort Dauerpatient mit nur ungefähren Symptomen zu werden. Joachim hat wie gesagt eine »feuchte Stelle« in der Lunge, die er auskurieren soll, ehe er als Fahnenjunker in sein Regiment eintritt. Und dieser stille, dieser in Wahrheit einfache und keusche junge Mensch ist es, den man als einzigen den Versuchungen des "Zauberbergs « widerstehen sieht, sowohl den sexuellen als denen zur Fahrlässigkeit mit Zeit, Pflicht und den sittlichen Aufgaben der Mannesarbeit. Er hat auch die Selbstbezwingung abzureisen, sogar gegen den Rat der Ärzte. Alle empfinden die schlichte Lauterkeit dieses Mannes. Aber er muß wiederkommen, den geliebten Beruf verlassen und eine "Nachkur» versuchen, während welcher Kehlkopfschwindsucht sich bei ihm einstellt und seinen frühen Tod verursacht. Mit ganz wenigen, leisen Linien, in einer Graphik möchte ich sagen, die alles Überflüssige ausspart, ist die Zeichnung dieses Charakters durchgeführt. Und sein Sterben gehört zu den ergreifendsten Schilderungen, die Mann je geglückt sind. Mag er überall in diesem Werk der Beobachter von unerhörtem Scharfblick, der Psycholog von tiefgründigsten Erkenntnissen, der Sprachkünstler ohnegleichen, der Dialektiker von blendender Unerschöpflichkeit, der Inventarist der entsetzlichen Leidensformen sein - hier ist er der Dichter, der zu erschüttern weiß, dessen Herz man klopfen hört! -

Ja, und als der Donnerschlag den Erdball erbeben macht, ist der arme Joachim schon tot. Der Kriegsausbruch reißt auch Hans Castorp vom Zauberberg hinab ins Flachland und im Trubel des Bahnhofs, da alles was deutsch heißt, hinabjagen will, sagt der arme Settembrini mit seinen schon hinschwindenden Kräften liebevoll dem jungen Freund Lebewohl. Es war für Mann nicht leicht, diesen jähen Schluß, gerade diesen, dem Werk anzuhängen. Er hat versucht, es mit Schlichtheit zu tun. Und in einer kurzen Kriegsszene sehen wir dann Hans Castorp sich ins Ungewisse verlieren. Zum Leben? Kaum. Zum Tod? Wahrscheinlich.

Ich sehe mit Schrecken, daß bei aller verhältnismäßigen Ausführlichkeit - für ein Referat ausführlich - ich nicht den dritten Teil der Notizen aufgearbeitet habe, die ich mir während des Lesens machte. Und mit dieser Feststellung allein bezeuge ich schon den unerhörten Reichtum des Inhaltes. Diejenigen, die durchaus ein Schlußurteil haben wollen, kann ich nur darauf verweisen, sich selbst aus allem Gesagten eines wenigstens ungefähr zu bilden. Wenn sie dann annehmen, daß hier bedeutendster 
geistiger Gewinn sich nur erobern läßt, durch Überwindung abschreckender stofflicher Materien, so werden sie auf der richtigen Spur sein. Daß der Verfasser selbst dafür eintritt - durch seinen Settembrini - daß das Pathologische recht eigentlich der Stoff für den Dichter sei, »da der schöne Geist sich fast regelmäßig das Leiden zum Gegenstand gesetzt hat « - wirkt als Verteidigung. Gewiß - das Leiden! Das der Seele, und sparsam und im Einzelfall das des Leibes. Aber zwischen den Schwären des armen Heinrich, der Wunde des Amfortas und einem ganzen Sanatorium voll Tuberkelnkranker ist ein Unterschied. 